\title{
Matrix-Metalloproteinases as Imaging Targets for Inflammatory Activity in Atherosclerotic Plaques
}

\author{
Michael Schäfers ${ }^{1}$, Otmar Schober ${ }^{2}$, and Sven Hermann ${ }^{1}$ \\ ${ }^{I}$ European Institute for Molecular Imaging-EIMI, Münster, Germany; and ${ }^{2}$ Department of Nuclear Medicine, \\ Westfälische Wilhelms-Universität Münster, Münster, Germany
}

\begin{abstract}
Cardiovascular events such as myocardial infarction or stroke resulting from atherosclerosis still account for the majority of deaths worldwide. New imaging approaches focusing on the visualization of inflammation in the vessel wall may emerge as tools for individualized risk assessment and prevention of events. Enzymes such as matrix-metalloproteinases (MMPs) are involved in several steps in plaque progression driving plaques into vulnerable, rupture-prone states. Targeting of MMPs for imaging is therefore a promising strategy. The rationale, potential, and current status of imaging MMPs in the clinical context of stroke and myocardial infarction are reviewed here from a clinical viewpoint.
\end{abstract}

Key Words: clinical cardiology; molecular imaging; vascular; PET; SPECT; atherosclerosis; matrix-metalloproteinases; plaque imaging

J Nucl Med 2010; 51:663-666

DOI: 10.2967/jnumed.109.065698

Vascular integrity warranting blood supply to organs is of crucial importance, especially in the case of the heart or brain. Although preventive measures on a population level and curative medicine have contributed to the continuing decline in age-adjusted cardiovascular mortality over recent years, early identification of the individual at risk of cardiovascular complications remains a great challenge. Cardiovascular events are responsible for over $50 \%$ of all deaths in Europe (European Heart Network, 2008; www. ehnheart.org), the United States, and many other developed or developing societies. Prevention of cardiovascular events is therefore urgently needed and is one of the major recent challenges of medicine. New molecular imaging approaches featuring the assessment of inflammatory processes in the vascular wall on top of existing anatomic and functional

\footnotetext{
Received Sep. 11, 2009; revision accepted Nov. 24, 2009.

For correspondence or reprints contact: Michael Schäfers, European Institute for Molecular Imaging-EIMI, Mendelstrasse 11, 48149 Münster, Germany.

E-mail: schafmi@uni-muenster.de

COPYRIGHT @ 2010 by the Society of Nuclear Medicine, Inc.
}

vessel imaging procedures could emerge as decisive tools for the understanding and prevention of cardiovascular events.

\section{INFLAMMATION, A HALLMARK OF ATHEROSCLEROTIC PLAQUES}

The main pathologic feature of atherosclerosis is the atherosclerotic lesion. It develops in the course of a series of highly specific cellular and molecular inflammatory responses of the vessel wall to an initial injury. The initial lesion, primarily causing endothelial dysfunction, can be triggered by various conditions such as hypercholesterolemia, hypertension, and smoking. The endothelium tries to neutralize this initial endothelial damage; if this is unsuccessful, chronic inflammation of the vessel wall results. The inflammation is characterized and driven by infiltration of cells (leukocytes, lymphocytes, macrophages, smooth muscle cells) into the vessel wall in conjunction with a concomitant incorporation of lipids. From the reversible fatty-streak state, the lesion can progress to a complex lesion (plaque) composed of activated macrophages, foam cells, T lymphocytes, mast cells and other such cells around a necrotic lipid-rich core. The prothrombotic content of the plaque is separated from the bloodstream by a thin fibrous cap. Although the atherosclerotic vessel wall can show outward remodelling partly compensating for narrowing of the vessel lumen by the plaque, luminal stenosis will eventually occur, resulting in poststenotic ischemia and the first clinical symptom (angina pectoris). The much more dangerous type of atherosclerotic lesion is unstable or vulnerable plaque, which can suddenly rupture and expel its thrombogenic content into the bloodstream, triggering a thrombotic vessel occlusion and the clinical sequela myocardial infarction or stroke. These plaques are often of lesser stenotic severity and thus will sometimes not impair blood flow at rest or even during exercise. At autopsy, they are characterized by a thin fibrous cap overlying cell-rich regions with a central core of extracellular lipid. Rupture of those plaques occurs mainly in their shoulder, where active macrophages accumulate and apoptosis may occur. The activated macrophages and mast cells locally destabilize the plaque 
shoulder by secreting a variety of matrix-degrading proteolytic enzymes such as matrix-metalloproteinases (MMPs) $(1,2)$. Although imaging of macrophages can already be performed today by ${ }^{18} \mathrm{~F}$-FDG PET, the specific advantage of the MMP imaging approach should be the assessment of the degrading activity of these macrophages mediated by MMPs finally resulting in plaque instability and rupture.

\section{MMPS AS MOLECULAR TARGETS FOR PLAQUE IMAGING}

MMPs are a group of at least 25 structurally related proteases that share a $\mathrm{Zn}^{2+}$-based catalytic mechanism. Extracellular matrix proteins and cell surface proteins are among substrates of MMPs. The majority of MMPs are excreted, only a minority of the known MMPs are bound to the cell surface. In tissues, MMP activity is counterbalanced by tissue inhibitors of MMPs (TIMPs); their presence prevents excessive proteolytic activity in physiologic states. MMPs can be clustered into different groups according to their substrate specificity and structural homology: interstitial MMPs such as collagenases (MMP-1, -8, and -13), gelatinases and basement membrane-degrading MMPs (MMP-2 and -9), stromelysins or matrilysins (MMP-3 and -7), membrane-bound MMPs (MMP-14 to -17), and others (MMP-12). MMPs are involved in physiologic processes such as organ development; however, disbalanced MMP activity is also known to be involved in different diseases in adult organisms. For example, tumors produce MMPs that cleave collagen, enabling growth of the tumor mass and metastases (3).

In a normal artery, pro-MMP-2, TIMP-1, and TIMP-2 that are nicely balanced can be detected. However, in vascular pathologies such as atherosclerosis, the MMP-TIMP balance gets out of control and MMP activity can be dramatically elevated. MMP overactivity is then driving plaque progression and destabilization, finally-and what is clinically most relevant-leading to plaque rupture and the clinical sequela myocardial infarction or stroke. Highly inflamed atheromatous plaques show increased global MMP activity (4); interstitial collagenases and gelatinases, especially, are significantly more highly expressed and activated in atheromatous (vulnerable) than in fibrous (stable) human plaques $(5,6)$. Although MMPs are expressed predominantly in macrophages, other cells such as vascular smooth muscle cells, lymphocytes, and endothelial cells may also produce MMPs (7). Most MMPs, except MMP11 and membrane-type MMPs, are secreted as inactive proforms and activated extracellularly.

MMPs are already involved in early phases of atherosclerosis, when the endothelium is damaged in response to different stresses. In the resulting inflammatory cascade, monocytes and macrophages stick to the injured endothelium. These abundantly produce and activate MMP-9 to facilitate their entry through the endothelial cell layer by degrading the basement membrane. Together with the concomitant ingestion of cholesterol, the plaque formation in the vessel wall is initiated and the plaque grows. The resulting reversible state is termed fatty-streak lesion. When cells enter the subintimal space, MMP activity is further boosted by inflammatory cytokines such as interleukin-1, tumor necrosis factor- $\alpha$, and oxidized low-density lipoprotein. In the course of further progression toward nonreversible states and complex and unstable lesions, MMPs are again involved and can have ambivalent functions. Degradation of the extracellular matrix by MMPs can thin the fibrous cap and reduce collagen content-typical features of vulnerable plaques prone to rupture (8). MMPs also promote plaque angiogenesis, another feature associated with vulnerable plaques (9). Facilitation of cell entry, destruction of extracellular matrix protein, thinning of the fibrous cap, and angiogenesis mediated by MMPs together seem therefore crucial for the progression of plaque toward vulnerable, high-risk lesions.

The local distribution of MMPs in atherosclerotic plaques further points toward their involvement in plaque destabilization: High MMP activity can be found in the cap and core area of the atherosclerotic plaque. For example, MMP1 overexpression occurs in areas of the fibrous cap with increased circumferential tensile stress, thus forming a predilection site for rupture. MMP activity can be further derived from collagen breakdown products abundantly observed in lipid-rich atherosclerotic plaques as evidence for enhanced extracellular matrix degradation (5). Besides local expression in plaques, circulating MMPs can also be found systemically in patients with acute myocardial infarction and unstable angina and are related to cardiovascular mortality (10). However, the source of these circulating MMPs-ruptured plaques versus myocardial remodeling - is not yet fully understood.

On the other hand, MMPs produced by endothelial and vascular smooth muscle cells could potentially stabilize plaques. Gelatinases and membrane-bound MMPs, involved in processes driving the plaque toward instability, also promote detachment of smooth muscle cells from their basement membranes and their migration and proliferation, processes that potentially thicken the fibrous cap and thereby stabilize atherosclerotic plaques (11).

In summary, MMP activity in atherosclerotic plaques is complex and can either drive plaque progression toward instability or stabilize plaques (Fig. 1). The time course of MMP expression and activation in individual plaques in mice and humans and the impact of emerging therapies on MMP activity (e.g., statins) are currently not known. Noninvasive molecular imaging modalities for MMP activity would ideally provide this information, both enhancing the understanding of plaque pathophysiology and assessing inflammatory plaque activity in patients.

\section{PRINCIPAL TARGETING STRATEGIES AND STATE OF THE ART OF NONINVASIVE IMAGING OF MMPS}

Targeting of MMPs for imaging can rely on different designs of MMP-assessing probes, which will be outlined 


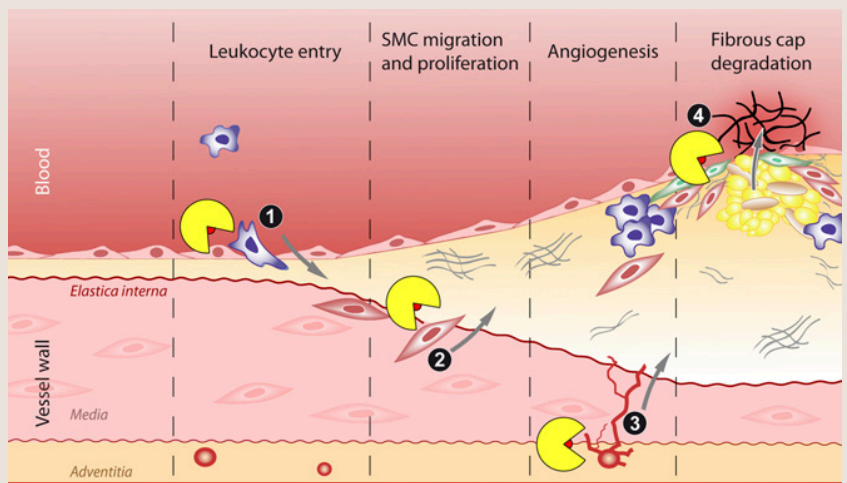

FIGURE 1. Impact of MMP activity on molecular and cellular pathways driving progression of atherosclerotic plaques. MMPs (yellow "Pac-Man") enable inflammatory cell infiltration into vessel wall (1), detachment and migration of smooth muscle cells from media (2) into fibrous cap and differentiation into fibroblasts, triggering of angiogenesis (3) resulting in sprouting of new vessels from vasa vasorum, and degradation of fibrous cap (4). Latter seems to be decisive step in transformation of plaque from stable to unstable, rupture-prone state.

here (Fig. 2). In principle, all strategies should focus on a high affinity and specificity toward MMPs, subtype selectivity, a favorable biodistribution, and low toxicity. As a prerequisite for the design of MMP imaging probes, the principal structure of MMPs is important. All MMPs have a conserved structural topology with a catalytic domain that includes 3 histidines complexing the zinc ion of the active site. Only activated MMPs expose the zinc ion; the zinc ion
A
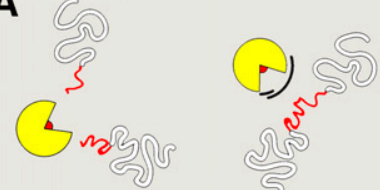

C

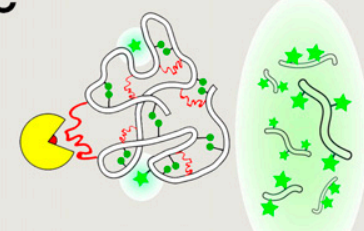

B

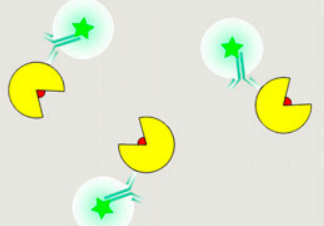

D

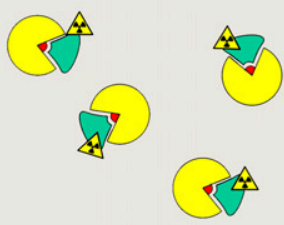

FIGURE 2. Strategies for targeting MMPs. (A) MMP enzyme (yellow "Pac-Man") with zinc ion (red). In activated state, substrates can bind to active site of enzyme and are cleaved (left); in inactivated MMP, zinc is not accessible (right). (B) MMP targeting by fluorescent antibodies. (C) MMP substrates housing quenched fluorochromes (left) emit light only when cleaved (right). (D) Synthetic MMP inhibitors bind active site with high affinities and can be labeled with isotopes or fluorescent dyes. is not accessible in MMP proenzymes or inactivated MMPs. Designing binders to the zinc ion is therefore a valuable strategy to differentiate between active and inactive MMPs.

\section{Antibodies}

The first attempt to visualize enzymes typically involves the design and application of antibodies. This is also true for MMPs, for which a variety of antibodies have been developed and are routinely applied for immunohistochemistry of MMP expression on histologic samples. In contrast to other strategies, these antibodies do not target the zinc ion of the MMP but rather bind to other epitopes on the protein surface. Therefore, antibodies usually cannot differentiate between active and inactive MMPs and are thus not suited as a principal strategy toward imaging of MMP activity.

\section{Substrates}

In contrast to antibodies, substrates specifically interact with the active site of the MMPs and are cleaved only by active enzymes. Furthermore, specific substrates for the different MMP subtypes exist, uniquely enabling selective MMP targeting. This approach was used in one of the first attempts to image MMP activity in vivo. Bremer et al. successfully used MMP substrates as smart probes for optical imaging of tumor-associated MMP activity. The developed imaging probe contains an MMP-2 peptide substrate with quenched near-infrared fluorochromes. In its unprocessed form, this probe is designed so that signals from the closely positioned fluorochromes are quenched. Only when the probe is cleaved are the fluorochromes enabled to emit light in the near-infrared range when excited. A unique advantage of this approach is that the resulting fluorescence signal is amplified, since one enzyme can cleave multiple probes. In mice bearing breast carcinomas, the authors were able to show that an intense accumulation of the fluorescent (i.e., cleaved) probe in MMP-positive tumors was diminished after treatment with an MMP inhibitor (12).

\section{Inhibitors}

A large variety of synthetic MMP inhibitors with high MMP affinity have been developed for therapeutic applications in tumors. However, most of these pharmaceuticals failed in clinical trials because of low therapeutic efficiency and significant side effects. In contrast to substrates, in which selectivity toward specific MMP subtypes can in principle be achieved, most of the existing MMP inhibitors have a broad range of affinities toward several MMP subtypes (3). This may in principle be a limitation of the approach with respect to the specificity of the resulting signal. In contrast to substrates, inhibitors bind to the enzyme in a 1:1 fashion, thereby providing no signal amplification. However, our group has successfully established 2 structurally distinct nonpeptidic MMP inhibitor-based probes (hydroxamates vs. barbiturates) for assessing MMP activity, which are labeled with $\gamma$-emitters for SPECT $\left({ }^{123} \mathrm{I}\right)$, positron emitters for PET 
FIGURE 3. In ApoE mouse kept on hypercholesterol diet for $20 \mathrm{wk}$, imaging of MMP activity in atherosclerotic plaques $2 \mathrm{~h}$ after injection of ${ }^{18} \mathrm{~F}$-labeled MMP inhibitor. Image is fused ex vivo PET and CT scans, with significant MMP activity (yellow) projected onto rendered high-resolution CT scan of arterial vascular tree prepared with a dedicated contrast agent (MicroFil; Flow Tech, Inc.). Images were generated using dedicated volumerendering software (www.voreen.org) jointly developed at University of Münster (20).

$\left({ }^{18} \mathrm{~F},{ }^{124} \mathrm{I}\right)$, or the fluorescent dye Cy5.5 for near-infrared fluorescence imaging (13-15). With this approach we were the first to show MMP activity in MMP-rich vascular lesions of the carotid artery in apolipoprotein E-deficient $\left(\mathrm{ApoE}^{-/-}\right)$ mice in vivo (Fig. 3), thereby proving the feasibility of using MMP inhibitors as molecular imaging probes (16). Meanwhile, other investigators have successfully used similar approaches to visualize MMP activity in atherosclerotic plaques in mice and rabbits and, most interestingly, to monitor responses to therapies such as lipid lowering by statins $(17,18)$. MMP inhibitors have not only been used for scintigraphic or fluorescence imaging but also were used in recent studies for coupling to MRI contrast agents such as gadolinium-1,4,7,10-tetraazacyclododecane- $N, N^{\prime}, N^{\prime \prime}, N^{\prime \prime \prime}$-tetraacetic acid to successfully image MMP activity in atherosclerotic plaques of $\mathrm{ApoE}^{-1-}$ mice in vivo (19).

\section{MMP IMAGING - WHEN WILL WE SEE IT IN THE CLINICS?}

Today, MMP imaging with either fluorescent substrates or labeled MMP inhibitors has been developed for preclinical applications and is successfully used in a variety of animal models of atherosclerosis. Although these models typically lack sufficient similarity to the human disease, preclinical studies show the feasibility of MMP imaging in vivo and form an excellent basis for proceeding toward clinical trials.

In patients, MMP imaging should focus primarily on studies on the pathophysiology to correlate the time course of MMP activation with the progression of atherosclerosis andmost interestingly - to correlate MMP activation with the clinical endpoints of infarction or stroke. If MMP activity proves to predict these events, MMP imaging could emerge as a decisive tool to assess cardiovascular risk in individual patients and prevent cardiovascular events.

\section{ACKNOWLEDGMENTS}

This work was partly supported by the Deutsche Forschungsgemeinschaft, SFB "Cardiovascular Molecular Imaging" projects $\mathrm{A} 2, \mathrm{C} 6$, and $\mathrm{Z} 2$, the Interdisciplinary Centre of Clinical Research (IZKF projects Schä2/020/09 and core unit SmAP), Münster, Germany, and by a research grant from Siemens Medical Solutions, Erlangen, Germany.

\section{REFERENCES}

1. Sanz J, Fayad ZA. Imaging of atherosclerotic cardiovascular disease. Nature. 2008;451:953-957.

2. Aikawa M, Libby P. The vulnerable atherosclerotic plaque: pathogenesis and therapeutic approach. Cardiovasc Pathol. 2004;13:125-138.

3. Wagner S, Breyholz HJ, Faust A, et al. Molecular imaging of matrix metalloproteinases in vivo using small molecule inhibitors for SPECT and PET. Curr Med Chem. 2006;13:2819-2838.

4. Choudhary S, Higgins CL, Chen IY, et al. Quantitation and localization of matrix metalloproteinases and their inhibitors in human carotid endarterectomy tissues. Arterioscler Thromb Vasc Biol. 2006;26:2351-2358.

5. Galis ZS, Sukhova GK, Lark MW, et al. Increased expression of matrix metalloproteinases and matrix degrading activity in vulnerable regions of human atherosclerotic plaques. J Clin Invest. 1994;94:2493-2503.

6. Sluijter JP, Pulskens WP, Schoneveld AH, et al. Matrix metalloproteinase 2 is associated with stable and matrix metalloproteinases 8 and 9 with vulnerable carotid atherosclerotic lesions: a study in human endarterectomy specimen pointing to a role for different extracellular matrix metalloproteinase inducer glycosylation forms. Stroke. 2006;37:235-239.

7. Newby AC. Matrix metalloproteinases regulate migration, proliferation, and death of vascular smooth muscle cells by degrading matrix and non-matrix substrates. Cardiovasc Res. 2006;69:614-624.

8. Johnson JL, Baker AH, Oka K, et al. Suppression of atherosclerotic plaque progression and instability by tissue inhibitor of metalloproteinase-2: involvement of macrophage migration and apoptosis. Circulation. 2006;113:2435-2444.

9. Pepper MS. Role of the matrix metalloproteinase and plasminogen activatorplasmin systems in angiogenesis. Arterioscler Thromb Vasc Biol. 2001;21:11041117.

10. Blankenberg S, Rupprecht HJ, Poirier O, et al. Plasma concentrations and genetic variation of matrix metalloproteinase 9 and prognosis of patients with cardiovascular disease. Circulation. 2003;107:1579-1585.

11. Newby AC. Metalloproteinases and vulnerable atherosclerotic plaques. Trends Cardiovasc Med. 2007; 17:253-258.

12. Bremer $\mathrm{C}$, Tung $\mathrm{CH}$, Weissleder R. In vivo molecular target assessment of matrix metalloproteinase inhibition. Nat Med. 2001;7:743-748.

13. Breyholz HJ, Wagner S, Levkau B, et al. A ${ }^{18} \mathrm{~F}$-radiolabelled analogue of CGS 27023A for assessment of matrix-metalloproteinase activity in vivo. $Q \mathrm{~J} \mathrm{Nucl}$ Med Mol Imaging. 2007;51:24-32.

14. Wagner S, Breyholz HJ, Law MP, et al. Novel fluorinated derivatives of the broad-spectrum MMP inhibitors $N$-hydroxy-2( $R$ )-[[(4-methoxyphenyl)sulfonyl](benzyl)- and (3-picolyl)-amino]-3-methyl-butanamide as potential tools for the molecular imaging of activated MMPs with PET. J Med Chem. 2007;50: 5752-5764.

15. Faust A, Waschkau B, Waldeck J, et al. Synthesis and evaluation of a novel fluorescent photoprobe for imaging matrix metalloproteinases. Bioconjug Chem. 2008; 19:1001-1008.

16. Schäfers M, Riemann B, Kopka K, et al. Scintigraphic imaging of matrix metalloproteinase activity in the arterial wall in vivo. Circulation. 2004;109: 2554-2559.

17. Hartung D, Schäfers M, Fujimoto S, et al. Targeting of matrix metalloproteinase activation for noninvasive detection of vulnerable atherosclerotic lesions. Eur $J$ Nucl Med Mol Imaging. 2007;34(suppl):S1-S8.

18. Fujimoto S, Hartung D, Ohshima $S$, et al. Molecular imaging of matrix metalloproteinase in atherosclerotic lesions: resolution with dietary modification and statin therapy. J Am Coll Cardiol. 2008;52:1847-1857.

19. Lancelot E, Amirbekian V, Brigger I, et al. Evaluation of matrix metalloproteinases in atherosclerosis using a novel noninvasive imaging approach. Arterioscler Thromb Vasc Biol. 2008;28:425-432.

20. Ropinski T, Hermann S, Reich R, et al. Multimodal vessel visualization of mouse aorta PET/CT scans. IEEE Trans Vis Comput Graph. 2009;15:1515-1522. 